\title{
25 Research Soure \\ Cell differentiation and aging is accompanied by depletion of the ACE2 protein
}

Eva Bartova ( $\sim$ bartova@ibp.cz)

Institute of Biophysics of Czech Acad. Sci. https://orcid.org/0000-0003-1299-0470

\section{Sona Legartova}

Institute of Biophysics of Czech Acad. Sci.

Jana Krejci

Institute of Biophysics of Czech Acad. Sci.

Orazio A. Arcidiacono

Institute of Biophysics of Czech Acad. Sci.

\section{Research Article}

Keywords: ACE2, renin, embryonic heart, lung cancer cells, human kidney embryonic cells, human colon adenocarcinoma cells, drug treatment, SARS-CoV-2

Posted Date: June 30th, 2020

DOI: https://doi.org/10.21203/rs.3.rs-39062/v1

License: (1) (1) This work is licensed under a Creative Commons Attribution 4.0 International License. Read Full License 


\section{Abstract}

Angiotensin-converting enzyme (ACE) is a zinc metalloproteinase involved in the renin-angiotensin system (RAS). It is well known that ACE and ACE2 are central regulators of blood pressure. Moreover, recently, it was observed that the ACE2 protein is the main target of the SARS-CoV-2 virus, so we have tried to reveal if there is a distinction in the levels of the ACE2 protein in distinct cell types (sensitive to virus infection), during cell differentiation and aging. We observed that depletion of the ACE2 protein appears in aorta-associated parts during the aging of adult mice, and the level of ACE2 was lowest in kidneys of old female animals in comparison to male mice. Differentiation into enterocytes and more pronouncedly into cardiomyocytes was accompanied by depletion of the ACE2 protein. The deficiency of histone deacetylase 1 (HDAC1) also caused a decrease in the level of both ACE2 and its interacting partner renin. However, experimental cardiomyogenesis was associated with renin up-regulation. In human lung adenocarcinoma cells, vitamin D2, but not chloroquine, slightly increased the level of ACE2. Together, the higher level of the ACE2 protein appears in non-differentiated cells and tissue of young mice, in comparisons to terminally differentiated cells and old animals; thus, a higher level of the ACE2 protein, also seen after vitamin D2 treatment, seems to be a barrier against SARS-CoV-2, because it is known that tissues of young individuals are less sensitive to viral infection.

\section{Introduction}

It is well-known that ACE cleaves angiotensin I to form angiotensin II (Rice et al. 2004). ACE and ACE2, are considered as regulators of blood pressure; thus, to study the polymorphisms in these genes is demanding from the view of understanding of the pathophysiology of hypertension. The insertion/deletion (I/D) polymorphism of the ACE gene (mapped on chromosome 17q23) and single nucleotide polymorphism G8790A of the ACE2 gene (mapped on chromosome Xp22) were found to be main pre- dispositions for systemic arterial hypertension (Chung et al. 2013). A co-regulatory function between ACE and ACE2 was found in the case of vasoconstriction and vasodilatation that proceeds in the heart and kidney while in the kidney, levels of the ACE2 protein decrease in experimental animals with hypertension. A similar phenomenon was observed in diabetic and pregnant rats (Burrell et al. 2004; Danilczyk and Penninger 2006).

Conversely, the up-regulation of ACE2 was observed in failing hearts (Wang et al. 2012), which documents that the ACE2 gene and its product the ACE2 protein play a regulatory role in the physiology of the heart, blood vessels, and kidneys. Thus, to understand the precise function of ACE2 could help to improve the treatment of pathophysiological states. Moreover, the ACE2 protein has multiple interacting partners, including renin playing also a role in the regulation of not only renal functions but also blood pressure (Fig. 1A, Danilczyk and Penninger 2006; Koitka et al. 2008). 
Interestingly, the high level of ACE2 can be observed in patients with diabetes mellitus that is treated by ACE inhibitors and blockers of the angiotensin II type-I receptor (ARBs). The ACE2 level can also be increased by the analgesic and non- steroidal anti-inflammatory drug (NSAID) ibuprofen, similarly, as it was observed in patients who have diabetes, treated with ACE inhibitors (Fang et al. 2020). Consequently, it is believed that the increased expression of ACE2 would facilitate infection with pathogenic coronaviruses SARS-CoV ( $\mathrm{Li}$ et al. 2003). Thus, it is likely that diabetes and hypertension, cured by ACE inhibitors, are comorbidities contributing to the unfavorable progression of the COVID-19 infection http://www.who.int/news-room/commentaries/detail/covid-19-and-the-use-of-angiotensin-convertingenzyme- inhibitors-and-receptor-blockers.

It is well-known that the genome of coronaviruses encodes the following proteins: the spike (S) protein, nucleocapsid $(\mathrm{N})$ protein, membrane $(\mathrm{M})$ protein, and the protein of the envelope $(\mathrm{E})$. The $S$ protein binds to ACE2 to invade the virus into the cell (Wan et al. 2020). Li (2016) showed that the SARS-CoV-2 virus invades the cells via a short intracellular tail, a transmembrane anchor, and a large ectodomain consisting of receptor binding S1 subunit and a subunit, called membrane-fusing S2. The findings that SARS-CoV-2 enters into the cells via the ACE2 receptor, open an avenue for the effective therapies for COVID-19 infection. It is likely and must be tested that anti-ACE2 antibodies could prevent SARS-CoV-2 binding to the ACE2 receptor.

Based on the data mentioned above, we studied the cellular distribution pattern and cellular levels of the ACE2 protein, in parallel with its interacting partner, renin. This approach has not been applied in any other analysis studying the function of ACE2. We hope that our results could be useful for the understanding of the ACE2 role in SARS-CoV-2 pathogenesis; and, thus, COVID-19 progression. We analyzed the changes of ACE2 cellular distribution, the level, and the function in the cell lines (or tissues) established from the lung, kidneys, hearts, and intestine of human and mouse origin, as well as we studied the changes in the ACE2 level during experimentally-induced mouse cardiomyogenesis in mouse embryonic stem cells ( $m E S C s$ ). These cells and mouse heart tissue were experimentally affected by compounds, promising from the view of COVID-19 treatment, including vitamin D2, chloroquine diphosphate, and selected ACE inhibitor. In such an experimental model, we anticipated which druginduced changes and cell differentiation-related changes in the levels of the ACE2 protein are likely adverse and/or positive factors for SARS-CoV-2 binding to the ACE2 protein in various tissues.

\section{Material And Methods}

\section{Cell cultivation and treatment}

Human lung cancer A549 cells and human adenocarcinoma HT29 cells were cultivated in DMEM (Dulbecco's Modified Eagle's Medium, Sigma Aldrich, Czech Republic)) supplemented with $10 \%$ fetal calf serum (FCS) (Merck, Germany). A549 lung cancer cells were treated by PARP inhibitor, olaparib (final concentration $10 \mu \mathrm{M}$ for 24 hours; \#S1060, Selleckchem, Germany), an inhibitor of histone deacetylase, SAHA (final concentration $5 \mu \mathrm{M}$ for 24 hours; \#10009929, Cayman Chemical Company, USA). Also, A549 
cells were treated by antimalarial chloroquine diphosphate (final concentration $16 \mu \mathrm{M}$ for 24 hours, \#S4157, Selleckchem, Germany), ACE inhibitor lisinopril (100 nM for 24 hours; \#S2076, Selleckchem, Germany), vitamin D2 (100 nM for 24 hours; \#S4035, Selleckchem, Germany), or by combination chloroquine diphosphate (16 $\mu \mathrm{M}$ for 24 hours), vitamin D2 (100 nM for 24 hours) and ACE inhibitor (100 $\mathrm{nM}$ for 24 hours).

The human intestine adenocarcinoma (HT29) cells were treated by the inhibitor of histone deacetylases (HDACs), sodium butyrate (NaBt; final concentration 5mM; \#B5887, Sigma Aldrich, Czech Republic) inducing enterocytic differentiation for 48 hours treatment see Bartova et al. (2005). In these types of experiments, we studied the effect of histone hyperacetylation and cell differentiation on the level of the ACE2 protein.

We also study the ACE2 level in human embryonal kidney cells, HEK293, cultivated in 293SFM II (\#11686029, Thermo Fisher Scientific, Czech Republic) growth medium. This is a serum-free, protein-free medium for growth and transfection of the suspension-adapted human embryonic kidney (HEK) 293 cells. For the cultivation, the medium was supplemented by 10\% FCS (Merck, Germany).

For irradiation by cobalt-60, cells were cultivated on Petri dishes, irradiated by the dose of 5 Gy of $\mathrm{Y}$-rays delivered by irradiation device Chisostat (the Chirana, Czech Republic). Cells were either fixed by $4 \%$ formaldehyde for immunostaining experiments or harvested for western blots 2 hours after $\mathrm{Y}$-irradiation.

\section{Cultivation of mouse ESCs and differentiation into cardiomyocytes}

Were studied following mESC lines: wild-type mESCs, D3 line (mESCs wt, wild-type), and mESCs that were characterized by histone deacetylase 1 (HDAC 1) deficiency (HDAC1 dn mESCs) (Zupkovitz et al. 2010). Mouse ESCs were cultivated on $0.2 \%$ gelatine-coated Petri dishes (valid for wt-cells) or Matrigel (\#354277, Corning Incorporate, USA)-coated plastic dishes (valid for HDAC1dn-cells). Mouse ESCs were grown in Dulbecco's Modified Eagle Medium (DMEM, Sigma Aldrich, Czech Republic).

The medium was supplemented with penicillin and streptomycin, $0.1 \mathrm{mM}$ non-essential amino acids, 1 $\mathrm{ng} / \mathrm{ml}$ mouse leukemia inhibitory factor (LIF), $100 \mu \mathrm{M}$ mono-thioglycerol, and $15 \%$ fetal bovine serum (FBS). Differentiation into cardiomyocytes via the formation of embryonic bodies (EBs) was performed following Kudova et al. (2016) and Arcidiacono et al. (2018). Cells were cultured at $37^{\circ} \mathrm{C}$ in a humidified atmosphere containing 5\% CO2. Differentiation into cardiomyocytes was activated by cell seeding into ES culture media without leukemia inhibitory factor (LIF). In this step, we used the "hanging drop" method. In day 3 of cultivation, EBs were placed on new plastic dishes; in day 6, EBs were transferred to gelatine-coated culture dishes with DMEM/F12 (1:1) (\#11320-033, Sigma Aldrich, Czech Republic) supplemented with insulin, transferrin, and selenium (ITS-100x, \#41400-045, ThermoFisher Scientific, Czech Republic) (DMEM/F12-ITS). The differentiation medium (DMEM/F12-ITS) was changed every two days. The duration of the differentiation into cardiomyocytes was up to day 20 (dd20).

Tissue isolated from experimental animals. 
Explanted hearts from mouse strain C57BI6 were studied on the distribution pattern of the ACE2 protein and its interacting partner, renin. Mice were kept in a pathogen-free (SPF) animal facility (see description in Arcidiacono et al. 2018). For experiments, we needed the agreement of the Ethics Commission of the Ministry of Agriculture of the Czech Republic (protocol No.48/2016). After breading, embryos were explanted from female animals 15 days post-conception (e15), and embryonic hearts were treated by HDAC inhibitors (200 nM TSA, $16 \mu \mathrm{M}$ SAHA, and $15 \mathrm{mM}$ VPA) (more detail see Vecera et al. 2018). Except for embryonic and young, old female and male hearts, we additionally isolated adult mouse lungs, brains, and kidneys. By the use of western blots, we compared the level of the ACE2 protein in selected tissues.

\section{Cell culture immunostaining}

Immunofluorescence was performed following Legartova et al. (2019). Briefly, the cells were fixed in $4 \%$ formaldehyde (PFA) for $10 \mathrm{~min}$ at room temperature (RT), permeabilized with $0.2 \%$ Triton $X-100$ (\#194854, MP Biomedicals, USA) for 10 min and 0.1\% saponin (\#S7900, Sigma Aldrich, Czech Republic) for $12 \mathrm{~min}$. After that, the dishes were washed twice in phosphate-saline buffer saline for $15 \mathrm{~min}$. We used 1\% bovine serum albumin (BSA; \#A2153-506, Sigma Aldrich, Czech Republic) dissolved in 1x PBS as a blocking solution. Next, the samples were incubated in the blocking solution for one hour at room temperature and then washed in 1x PBS for $15 \mathrm{~min}$. For immunofluorescence analysis, the following antibodies were used: anti-ACE2 (\#ab15348, Abcam, UK), anti-renin (\#PA5-102432, ThermoFisher Scientific, Czech Republic) and a-actinin (\#A7811, Sigma Aldrich, Czech Republic). As secondary antibodies, we used: Alexa Fluor 594-conjugated goat anti-rabbit (\#A11037, ThermoFisher Scientific, Czech Republic), Alexa Fluor 594-conjugated goat anti- mouse (\#A11032, ThermoFisher Scientific, Czech Republic), Alexa Fluor 488- conjugated goat anti-rabbit (\#ab150077, Abcam UK). Negative control was considered samples incubated without primary antibodies. Cell nuclei (GC-rich sequences of DNA) were stained by 4',6-diamidino-2-phenylindole (DAPl; Merck, Czech Republic). As a mounting medium, we used Vectashield (\#H-1000, Vector Laboratories, USA).

\section{Heart tissue cryosectioning}

Fixed mouse hearts were frozen in embedding medium (OCT embedding matrix, Leica Microsystems, Mannheim, Germany) at $-80^{\circ} \mathrm{C}$. Mice hearts were sectioned using a Leica Cryo-microtome (Leica CM 1800, Leica, Germany). The cryo-sections were washed in PBS. The thickness of the sections was 13-14 $\mu \mathrm{m}$. For the immunofluorescence technique, we used the protocol published in Vecera et al. (2018). The primary antibody was anti-ACE2 (\#ab15348, Abcam, UK), as the secondary antibody we used Alexa Fluor 594-conjugated goat anti-rabbit (\#A11037, ThermoFisher Scientific, Czech Republic). DNA was counterstained using 4',6- diamidino-2-phenylindole (DAPI; Merck, Czech Republic), and the mounting medium was Vectashield (Vector Laboratories, Vector Laboratories, USA).

\section{The tile-scanning}

Cryo-sections of whole embryonic hearts (at embryonal stage e15) were stained by appropriate antibodies, as mentioned above. For analysis, we used the "tile-scanning" mode, a toll of Leica SP-5 
confocal microscope (Leica, Germany). To acquire images, we used HCX PL APO lambda blue 20.0× 0.7 IMM UV objective (Leica Microsystems, Germany) (see Bartova et al. 2016).

\section{Laser scanning confocal microscopy}

For analyses, we used a Leica TCS SP8-X SMD confocal microscope (Leica Microsystem, Germany), equipped with $63 \times$ oil objective (HCX PL APO, lambda blue) with a numerical aperture $(N A)=1.4$. Image acquisition was performed using a white light laser (WLL; wavelengths of 470-670 nm in $1 \mathrm{~nm}$ increments) with the following parameters: $1024 \times 1024$-pixel resolution, $400 \mathrm{~Hz}$, bidirectional mode, and zoom 8-12 using the Leica Application Suite (LAS X) software.

\section{Western blotting}

Western blots were performed following Jugova et al. (2011). Cell cultures or tissue samples were washed with PBS and lysed in sodium dodecyl sulfate (SDS) lysis buffer $(50 \times 103 \mathrm{~mol} / \mathrm{I} \mathrm{Tris-HCl,} \mathrm{pH} 7.5$; $1 \%$ SDS; $10 \%$ glycerol). The total protein concentration was determined by the DC protein assay kit (\#5000111, Bio-Rad, Czech Republic) and ELISA Reader $\mu$ Quant (BioTek, USA). The proteins were separated by SDS polyacrylamide gel electrophoresis (SDS-PAGE) and transferred to polyvinylidene difluoride (PVDF) membranes. The membranes were blocked with $2 \%$ fat-free milk or $2 \%$ gelatin for one $h$ and then immunoblotted overnight at $4^{\circ} \mathrm{C}$ with the following primary antibodies against the ACE2 protein (\#ab15348, Abcam, UK). Primary antibodies were diluted 1:1000, and as a secondary antibody, we used goat anti-rabbit IgG (\#AP307P, Merck, Czech Republic; 1:2000). The western blot data were normalized to the amount of total protein and were quantified by using ImageJ software (NIH freeware). The Student's ttest was used for statistical analysis (Sigma Plot software version 14.0, Jandel Scientific, USA). Statistical significance at $p \leq 0.05$ is shown by $\left(^{*}\right)$.

\section{Statistical analyses and quantification of fluorescence intensity}

For analysis of western blot fragment density and analysis of fluorescence intensity, we used ImageJ (NIH freeware) and ImageQuant TL software. For statistical analysis, we used the Student's t-test. Experiments were repeated trice.

\section{Results}

localization of the ACE2 protein in the cells.

The ACE2 protein is mainly located in the cytoplasm of the cells, but fluorescence signals we also observed in the nucleoplasm, as it is shown in lung carcinoma A549 cells (Fig. 1B). Also, we studied the density of the ACE2 protein in distinct regions of embryonic hearts (at stage e15, e.g., 15 days after postconception). We have analyzed the following parts in embryonic hearts: right atrium (RA), left atrium (LA), aorta (AO) and pulmonary trunk (PT), right ventriculus (RV), and left ventriculus (LV) or intraventricular septum (IVS). We observed a relatively homogeneous immunofluorescence signal for ACE2 studied in the 
heart sections. However, an exception should be considered the ACE2 level in the aorta and pulmonary trunk (blue ellipse in Fig. 1C).

The levels of the ACE2 protein in distinct cell types.

By western blots, we studied the levels of the ACE2 protein and its relationship to renin, a prominent ACE2-interacting partner (Fig. 1A, Fig. 2). The levels of both ACE2 and renin we have analyzed in following cell lines representing an example of tissue preferentially affected by SARS-CoV-2 virus: human lung adenocarcinoma A549 cells, human intestinal adenocarcinoma HT29 cells (non-treated and differentiated by HDAC inhibitor, sodium butyrate; NaBt), human embryonal kidney cells HEK293, and mouse embryonic stem cells (mESCs; line D3) differentiated into cardiomyocytes. We observed that experimentally-induced cardiomyogenesis is characterized by depletion of ACE2, but the renin level was increased (Fig. 2). Surprisingly, intestinal cells HT29 were characterized by an appearance of additional ACE2 fragment, which level was increased when the density of the main ACE2 fragment was slightly decreased by NaBt treatment initiating the differentiation of these cells into mature enterocytes (Fig. 2, Bartova et al. 2005). Considering that the highest level of the renin protein was in intestinal cells and lung carcinoma cells, but not in embryonic kidney HEK293 cells (Fig. 2).

We have also analyzed the ACE2 protein and renin in mouse hearts isolated from young and old adult male and female animals (Fig. 3A). Western blot analyses were performed at tissue isolated from ventricular parts of the heart, atrium, and in the region concerning aorta and pulmonary trunk (aortaassociated vessels). Interestingly, we observed that the level of ACE2 is highest in young female aorta regions in comparison to old female aortas, while in male aortas, the levels of ACE2 were identical when we compared young and old animals (Fig. 3A). When we studied the

ACE2 level in mouse brains, interestingly, the highest level of ACE2 was found in olfactory bulbs in comparison to hippocampi and the brain cortex. These results were, in many cases, identical in both genders as well as young and old animals (Fig. 3B). In adult mouse lungs, we observed a subtle level of the ACE2 (ACE2 was on the detectable level) in comparison to mESCs, used as reference samples (Fig. 3C). However, in adult mouse kidneys, the ACE2 level was very high, and it was higher in male animals when compared with female mice of the same age (Fig. 3D). Importantly, old female animals were characterized by the lowest level of the ACE2 protein (Fig. 3D). Generally, the comparison of young and old female samples (heart- Ao, brain - OB, HIP, CTX, and kidneys of female mice), showed a decrease of the ACE2 level during aging (Fig. 3A-D).

Lung carcinoma cells treated by vitamin D2 and ACE inhibitor Lisinopril. Experiments were performed in A549 lung cancer cells treated by vitamin D2 and lisinopril, which concentrations were optimized by western blots. For our analysis, we used a dose of $100 \mathrm{~nm}$ of vitamin D2 and $100 \mathrm{nM}$ of Lisinopril (Fig. 4). Also, we have studied the effect of PARP inhibitor (olaparib), HDAC inhibitor (SAHA), $Y$-irradiation, and antimalarial chloroquine diphosphate on the level of the ACE 2 protein and renin in lung carcinoma cells A549 (Fig. 4). We found only subtle changes caused by selected drugs' treatment. Especially treatment by vitamin D2 caused a slight increase in the level of the ACE2 protein. We addressed a question if there is 
an additional effect of, for instance, chloroquine, in which we expected potentiation of the effect of vitamin D2. However, a combination of vitamin D2 and chloroquine did not up-regulate the level of ACE2 (Fig. 4). Together, only vitamin D2 enhances the level of the ACE2 protein, which seems to be the benefit of this vitamin likely working against SARS-Cov- 2 infection (llie et al. 2020).

Down-regulation of ACE2 in HDAC1 wt and HDAC dn mouse embryonic stem cells undergoing differentiation into cardiomyocytes.

In non-differentiated mESCs, we observed a relatively high level of the ACE2 protein. Compared with HDAC1 (histone deacetylase 1) depleted cells, HDAC1 wt cells were characterized by a higher level of ACE2 (Fig. 5). Experimentally-induced differentiation into cardiomyocytes in both wt and HDAC1 dn cells was accompanied by down- regulation of ACE2 but upregulation of renin (Fig. 5). Similarly, when ACE2 level was higher in treated A549 cells, renin level was lower (Fig. 5); thus, it seems likely that the ACE2 protein and renin works in an antagonistic way (Fig 2 and 5). Importantly, HDAC1 depleted cells were characterized by a late onset of renin up-regulation during cardiomyogenesis; it was 15th (dd15) and 20th (dd20) day of differentiation, while in HDAC1 wt mESCs, renin started to be up-regulated at day 10th (dd10) of differentiation (Fig. 5). Together, we summarize that HDAC1 deficiency affects the levels of both ACE2 and its interacting partner renin.

Additionally, in wt and HDAC1 dn cells, we have also analyzed the distribution pattern of the ACE2 protein in the cytoplasm and the cell nucleus (Fig. 6A-D). In non- differentiated wt and HDAC1 dn mESCs, we observed a relatively low level of ACE2 in the cell nucleus, but a high density in the nucleoplasm, especially the cell surface. However, cell differentiation into cardiomyocytes significantly changes the Nucleo/ Cytoplasmic ratio (N/C) (Fig. 6E, F).

HDAC inhibitor TSA enhanced the level of ACE2 in explanted embryonic hearts.

We performed an analysis in explanted embryonic hearts treated by HDAC inhibitors TSA and SAHA, and we observed a pronounced up-regulation of the ACE2 protein in TSA-treated hearts. Interestingly, embryonic hearts were characterized by a significantly higher level of the ACE2 protein in comparison to explanted hearts from adult animals (Fig. 7). Thus, again, higher levels of ACE2 in embryonic hearts, when compared with adult tissue, imply that ACE2 up-regulation is a benefit of the organism that resists to the SARS-Cov-2 virus infection.

\section{Discussion}

Here we addressed a question of how compounds reducing SARS-CoV-2 infection and its progression affect the levels of the ACE2 protein and its interacting partner renin. Additionally, we discussed whether differentiation into cardiomyocytes and enterocytes is accompanied by changes in the ACE2 level. Also, we analyzed ACE2 and renin in distinct cell types that are considered to be the main targets of the SARSCov-2 virus. Studied cell lines or tissues originated from lungs, hearts, intestine, and kidneys of a mouse or human individuals. Analysis by western blots showed an enhanced level of ACE2 in A549 cells treated 
by vitamin D2 (Fig. 4), which fits well with data showing the benefit of ACE2 high level as, for example, published Smiths et al. These authors documented that tobacco increases the level of ACE2 which prevents the binding of S1 fragment of SARS-CoV-2 to the ACE2 receptor; thus, it prevents the COVID19 progression (Smith et al. 2020).

In addition, we observed that human intestinal cells, HT29, were characterized by an appearance of additional ACE2 fragment, which level was increased when the density of the main ACE2 fragment was reduced by NaBt treatment causing differentiation of HT29 cells into mature enterocytes (Fig. 2). The level of other protein, renin, was significantly high in these intestinal cells as well as lung carcinoma cells, in comparison to embryonic kidney cells, characterized by renin depletion (Fig. 2). This surprising fact can be explained by the observation of Shaw et al. (2002), showing that widely experimentally used HEK293 cells are characterized by unexpected features of neurons; thus, these cells are not typical kidney epithelial cells.

Here, we additionally found that differentiation into cardiomyocytes, as well as into enterocytes is accompanied by depletion of the ACE2 protein, so it fits well with a sensitivity of older adults to the SARSCoV-2 infection, because pronounced cell differentiation is in developing embryos and during young age, while aging is characterized by heterochromatinization processes, gene silencing, features of terminally differentiated cells and cells undergoing senescence. We showed that this process is characterized by a low level of ACE2, which we observed here when we compare embryonic hearts with hearts explanted from adult mice (Fig.3 and Fig. 7).

We observed ACE2 depletion in hearts and kidneys of old female mice; thus, it fits well with our suggestion that low level of ACE2 seems to be the pre-disposition for more aggressive infection by SARSCoV-2, while ACE2 high level likely prevents binding of this RNA virus to its target cellular molecules. In this line, Smith et al. (2020) measured the level of the ACE2 gene expression in various tissues. They found that women and men produced similar amounts of ACE2 inside their lung cells. They also couldn't find any differences between young adults and older ones. So, in their observations, aging didn't change the ACE2 profiles. But in smokers, it was different. They analyzed gene expression inside the lungs of smokers versus non-smokers, and they saw a considerable spike in ACE2 coming from one particular kind of cell: secretory goblet cells (Smith et al. 2020). In our case, we analyzed the heart tissue of young and old male and female animals. Surprisingly, in comparison to the young animal, we observed a depletion of the ACE2 protein in aortas of old female mice (Fig. 3A). The same trend we have found in mouse kidneys, characterized by a very high level of the ACE2 protein (in comparison to mouse hearts, brains, and lungs) and aging, was accompanied by ACE2 depletion in only female animals. This observation is a little bit contradictory to the claim that preferably male individuals are more prone to virus infection. However, Jin et al. (2020) showed that men and women have the same prevalence of the COVID-19 disease, but men with COVID-19 have a higher risk for worse outcomes and death, independent of age.

\section{Conclusions}


Together, our experimental data indirectly imply that the higher level of the ACE2 protein seems to be a barrier against SARS-CoV-2 infection. An increase in the ACE2 level was mostly accompanied by renin down-regulation. Moreover, vitamin D2 caused a slight up-regulation of ACE2. Another agent, including antimalarial chloroquine diphosphate and ACE inhibitor lisinopril, did not affect the level of ACE2, so additional compounds, like vitamin K, nicotine, and /or dexamethasone (Hartmann- Boyce et al. 2020, Horby et al. 2020,) should be furthermore tested from the view of their effect on the ACE2 levels. Also, this should be checked during cell differentiation processes and aging that, according to our data, cause ACE2 down-regulation.

\section{Declarations}

\section{Contribution of the authors}

EB wrote the paper and experimented for Fig. 1B, she designed experiments and wrote this paper. SL was responsible for immunofluorescence staining and western blots shown in Fig. 2, 4, and 5. OA performed western blots and immunofluorescence on explanted hearts and mouse embryonic stem cells (Figs 1C, 6, and 7). JK is responsible for animal breeding, heart explanation, and western blots on heart tissue isolated from young and old experimental animals (Fig. 3).

\section{Funding}

This work was supported by the Strategy of the Czech Academy of Sciences (program Qualitas, ICO 68081707).

\section{Conflict of interest statement}

The authors have declared that there are no conflicts of interest.

\section{References}

1. Arcidiacono OA, Krejci J, Suchankova J, Bartova E. 2018. Deacetylation of Histone H4 Accompanying Cardiomyogenesis is Weakened in HDAC1-Depleted ES Cells. Int J Mol Sci 19.

2. Bartova E, Pachernik J, Harnicarova A, Kovarik A, Kovarikova M, Hofmanova J, Skalnikova M, Kozubek M, Kozubek S. 2005. Nuclear levels and patterns of histone H3 modification and HP1 proteins after inhibition of histone deacetylases. J Cell Sci 118: 5035-5046.

3. Bartova E, Vecera J, Krejci J, Legartova S, Pachernik J, Kozubek S. 2016. The level and distribution pattern of HP1 beta in the embryonic brain correspond to those of H3K9me1/me2 but not of H3K9me3. Histochem Cell Biol 145: 447-461.

4. Burrell LM, Johnston Cl, Tikellis C, Cooper ME. 2004. ACE2, a new regulator of the renin-angiotensin system. Trends Endocrinol Metab 15: 166-169. 
5. Danilczyk U, Penninger JM. 2006. Angiotensin-converting enzyme II in the heart and the kidney. Circ Res 98: 463-471.

6. Fang Y, Guo Z, Chai Y, Zhang H, Wang Z. 2020. [2019 Novel coronavirus, renin- angiotension system imbalance and coronavirus disease 2019]. Zhonghua Wei Zhong Bing Ji Jiu Yi Xue 32: 613-617.

7. Hartmann-Boyce J, Morris E, Goyder C, Kinton J, Perring J, Nunan D, Mahtani K, Buse JB, Del Prato S, Ji L et al. 2020. Diabetes and COVID-19: Risks, Management, and Learnings From Other National Disasters. Diabetes Care.

8. Horby P, Lim WS, Emberson J, Mafham M, Bell J, Linsell L, Staplin N, Brightling C, Ustianowski A, Elmahi E et al. 2020. Effect of Dexamethasone in Hospitalized Patients with COVID-19: Preliminary Report; doi: https://doi.org/10.1101/2020.06.22.20137273.

9. Chung CM, Wang RY, Fann CS, Chen JW, Jong YS, Jou YS, Yang HC, Kang CS, Chen CC, Chang HC et al. 2013. Fine-mapping angiotensin-converting enzyme gene: separate QTLs identified for hypertension and for ACE activity. PLoS One 8: e56119.

10. Ilie PC, Stefanescu S, Smith L. 2020. The role of vitamin D in the prevention of coronavirus disease 2019 infection and mortality. Aging Clin Exp Res 32: 1195- 1198.

11. Jin JM, Bai P, He W, Wu F, Liu XF, Han DM, Liu S, Yang JK. 2020. Gender Differences in Patients With COVID-19: Focus on Severity and Mortality. Front Public Health 8: 152.

12. Jugova A, Sustackova G, Legartova S, Stixova L, Kozubek S, Bartova E. 2011. Effects of epigeneticbased anti-cancer drugs in leukaemia and multiple myeloma cells. Cell Biol Int 35: 1195-1203.

13. Koitka A, Cooper ME, Thomas MC, Tikellis C. 2008. Angiotensin converting enzyme 2 in the kidney. Clin Exp Pharmacol Physiol 35: 420-425.

14. Kudova J, Vasicek O, Ciz M, Kubala L. 2016. Melatonin promotes cardiomyogenesis of embryonic stem cells via inhibition of HIF-1alpha stabilization. J Pineal Res 61: 493-503.

15. Legartova S, Lochmanova G, Zdrahal Z, Kozubek S, Sponer J, Krepl M, Pokorna P, Bartova E. 2019. DNA Damage Changes Distribution Pattern and Levels of HP1 Protein Isoforms in the Nucleolus and Increases Phosphorylation of HP1beta- Ser88. Cells 8.

16. Li F. 2016. Structure, Function, and Evolution of Coronavirus Spike Proteins. Annu Rev Viro/ 3: 237261.

17. Li W, Moore MJ, Vasilieva N, Sui J, Wong SK, Berne MA, Somasundaran M, Sullivan JL, Luzuriaga K, Greenough TC et al. 2003. Angiotensin-converting enzyme 2 is a functional receptor for the SARS coronavirus. Nature 426: 450-454.

18. Rice GI, Thomas DA, Grant PJ, Turner AJ, Hooper NM. 2004. Evaluation of angiotensin-converting enzyme (ACE), its homologue ACE2 and neprilysin in angiotensin peptide metabolism. Biochem $J$ 383: 45-51.

19. Shaw G, Morse S, Ararat M, Graham FL. 2002. Preferential transformation of human neuronal cells by human adenoviruses and the origin of HEK 293 cells. FASEB J 16: 869-871. 
20. Smith JC, Sausville EL, Girish V, Yuan ML, Vasudevan A, John KM, Sheltzer JM. 2020. Cigarette Smoke Exposure and Inflammatory Signaling Increase the Expression of the SARS-CoV-2 Receptor ACE2 in the Respiratory Tract. Dev Cell 53: 514- 529 e513.

21. Vecera J, Bartova E, Krejci J, Legartova S, Komurkova D, Ruda-Kucerova J, Stark T, Drazanova E, Kasparek T, Sulcova A et al. 2018. HDAC1 and HDAC3 underlie dynamic H3K9 acetylation during embryonic neurogenesis and in schizophrenia-like animals. J Cell Physiol 233: 530-548.

22. Wan Y, Shang J, Graham R, Baric RS, Li F. 2020. Receptor Recognition by the Novel Coronavirus from Wuhan: an Analysis Based on Decade-Long Structural Studies of SARS Coronavirus. J Viro/94.

23. Wang W, Bodiga S, Das SK, Lo J, Patel V, Oudit GY. 2012. Role of ACE2 in diastolic and systolic heart failure. Heart Fail Rev 17: 683-691.

24. Zupkovitz G, Grausenburger R, Brunmeir R, Senese S, Tischler J, Jurkin J, Rembold M, Meunier D, Egger G, Lagger $S$ et al. 2010. The cyclin-dependent kinase inhibitor $\mathrm{p} 21$ is a crucial target for histone deacetylase 1 as a regulator of cellular proliferation. Mol Cell Biol 30: 1171-1181.

\section{Figures}
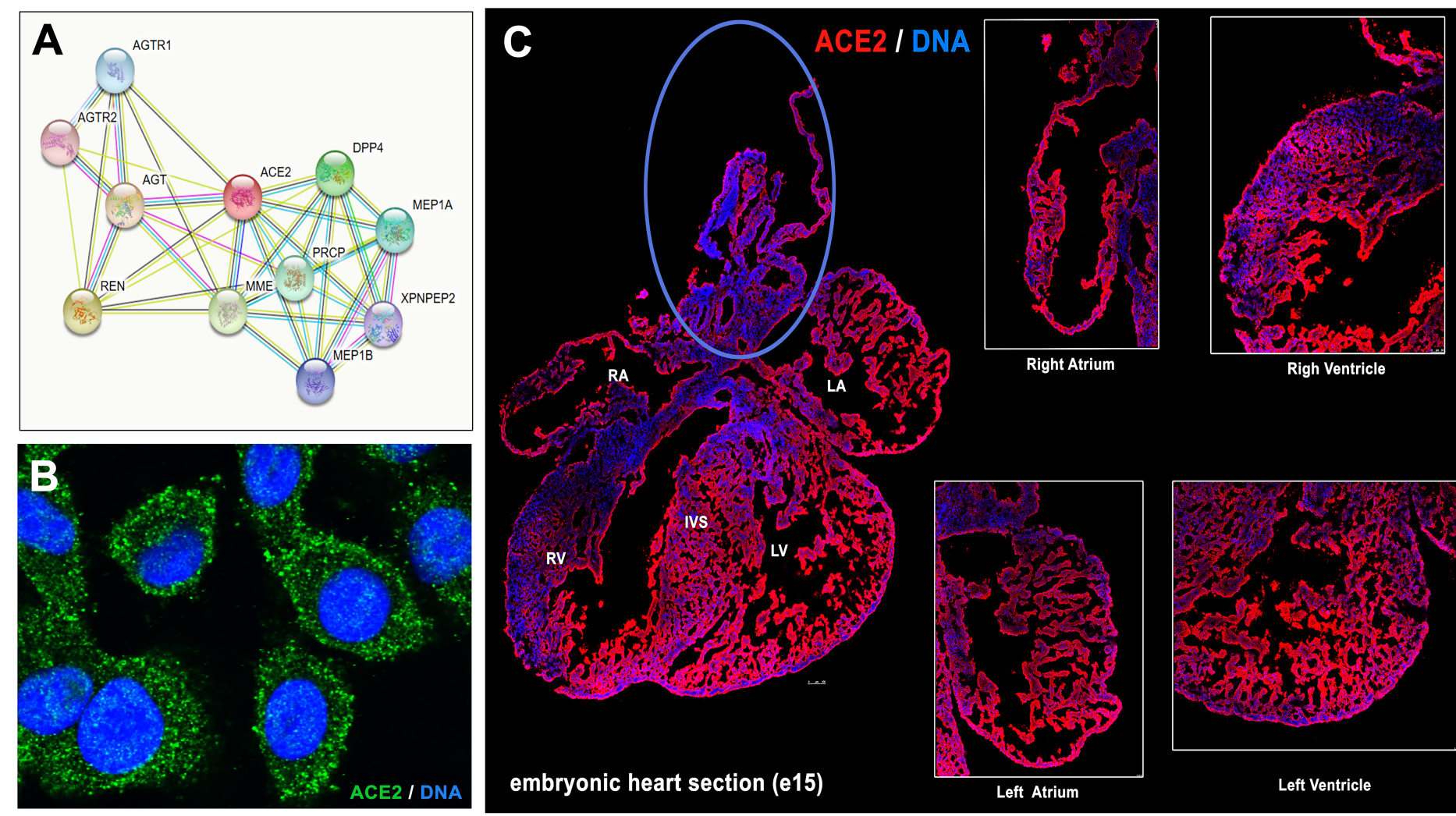

embryonic heart section (e15)
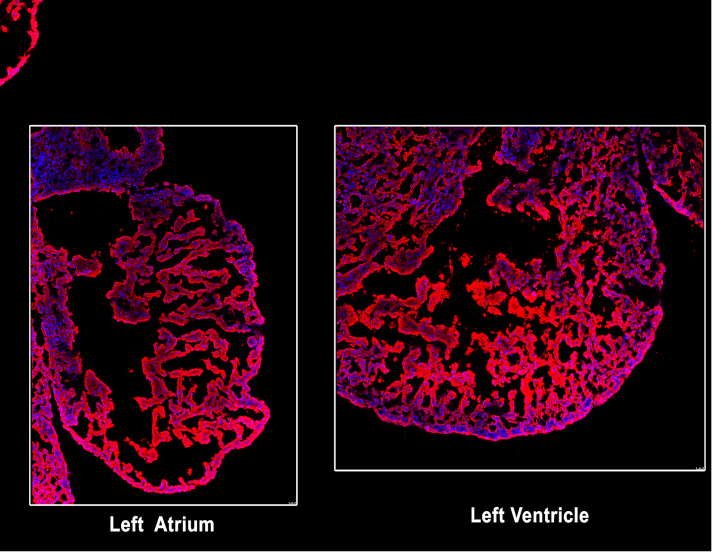

\section{Figure 1}

ACE2 in lung A549 cells and mouse embryonic heart section. (A) Interacting partners of ACE2 (https://string-db.org/network/9606.ENSP00000389326). (B) The ACE2 protein in A549 cells. (C) ACE2 in mouse heart section (right atrium (RA), left atrium (LA), aorta (AO) and pulmonary trunk (PT), right 
ventriculus (RV) and left ventriculus (LV) or intraventricular septum (IVS). The blue ellipse shows the anatomical part associated with the aorta.

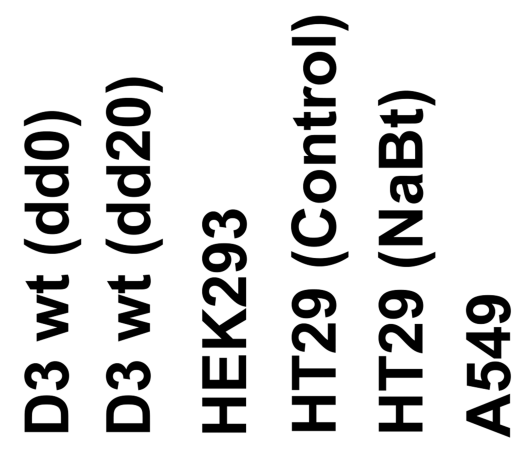

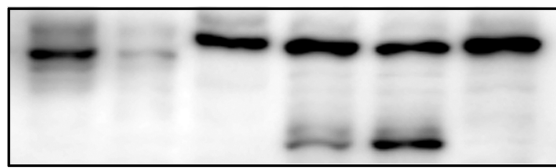

*
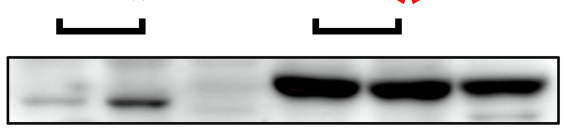

*

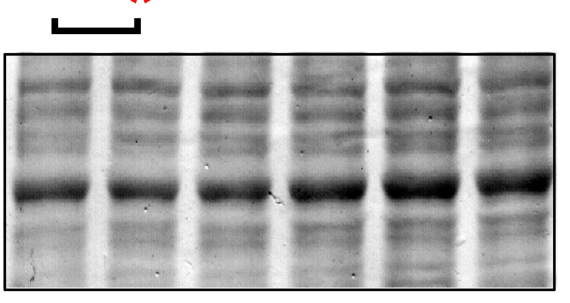

\section{Total protein \\ level}

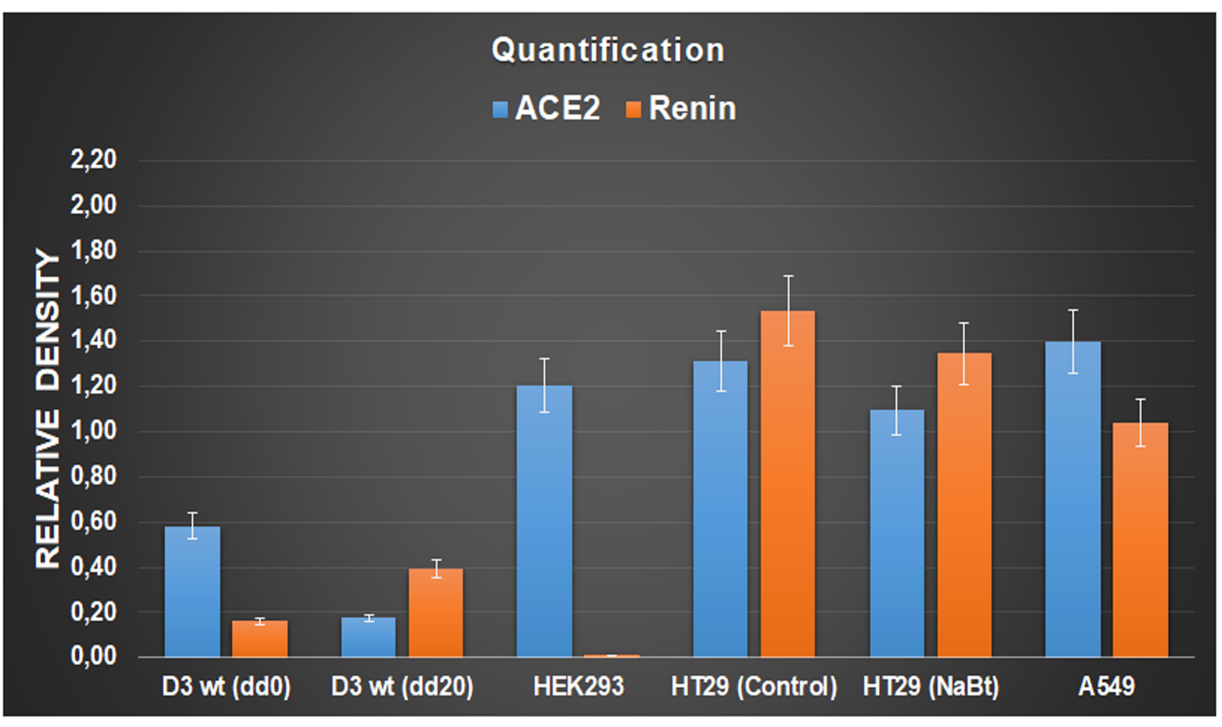

Figure 2

The levels of the ACE2 and renin in distinct cell types. The analysis was performed in mouse embryonic stem cells (mESCs) differentiated into cardiomyocytes, human embryonal kidney cells HEK293, human intestinal adenocarcinoma HT29 cells (non-treated and differentiated by HDAC inhibitor, sodium butyrate; 
$\mathrm{NaBt}$ ), and human lung adenocarcinoma A549 cells. Asterisks show a statistically significant change in the protein levels. Student's t-test was applied for the analysis.
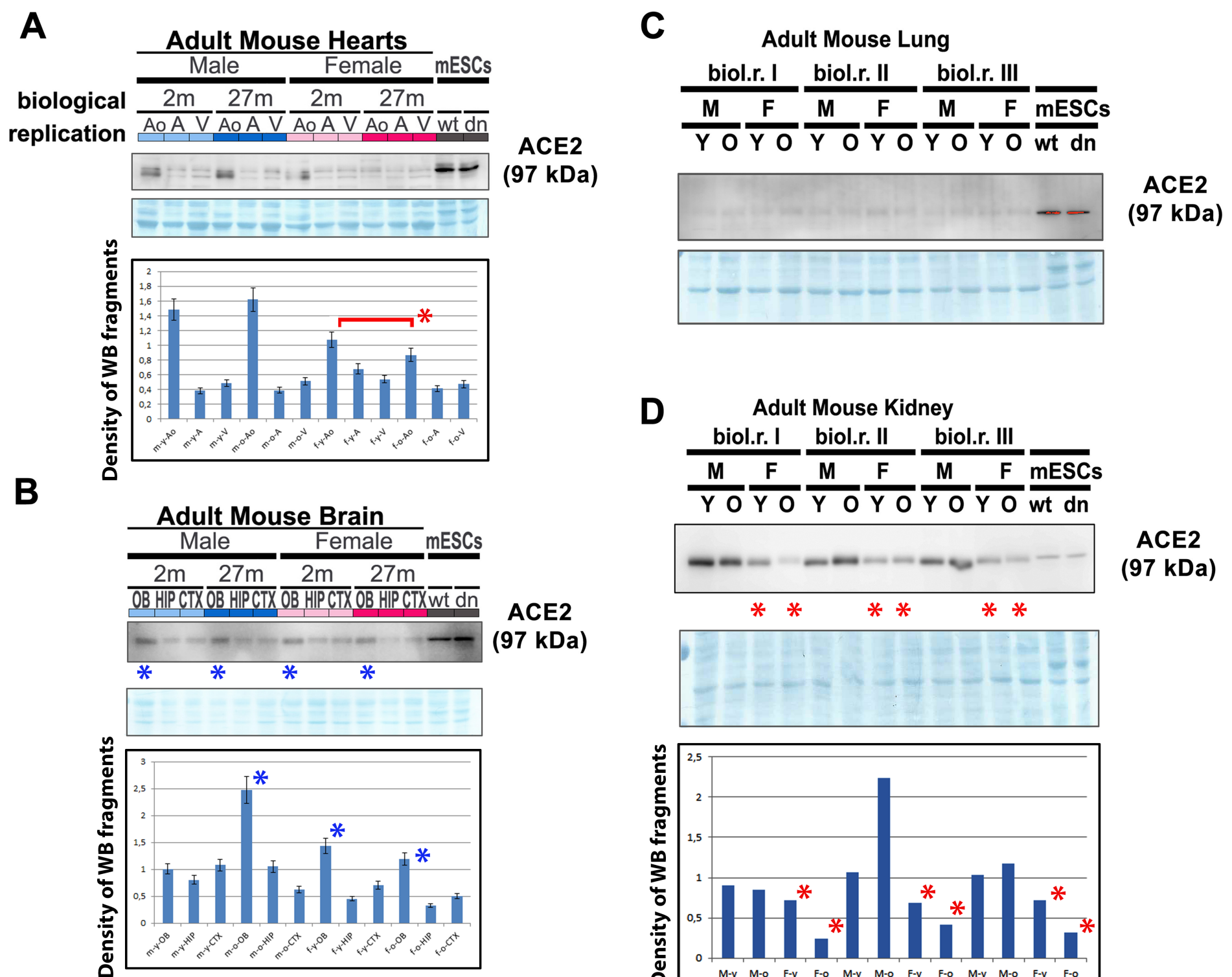

ACE2

(97 kDa)

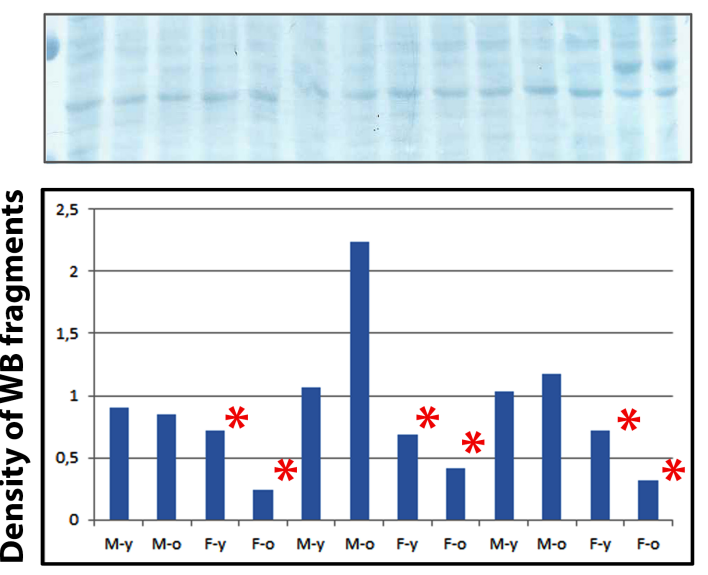

\section{Figure 3}

ACE2 and renin levels in mouse hearts, brains, lungs, and kidneys isolated from young and old animals. Adult mouse hearts were sectioned following the anatomy: Ventricular parts $(V)$, atrium $(A)$, and vessels associated with the aorta (Ao). The levels of selected proteins were studied by western blots, and data were normalized to the total protein levels. The level of the ACE2 protein was also studied in brains, lungs, and kidneys isolated from young and old male and female mice. Quantification was done by ImageJ software. Red asterisks mean a decrease in the protein level; blue asterisks show a high ACE2 protein level in comparison with other samples analyzed. 
A549

\begin{tabular}{cccccccccc}
1 & 2 & 3 & 4 & 5 & 6 & 7 & 8 & 9 & 10 \\
\hline- & - & - & - & - & - & - & - & - & - \\
\hline- & + & - & - & - & - & - & - & - & - \\
\hline- & - & + & - & - & - & - & - & - & - \\
\hline- & - & - & + & - & - & - & - & - & - \\
\hline- & - & - & - & + & - & + & - & - & + \\
\hline- & - & - & - & - & + & + & - & + & + \\
\hline- & - & - & - & - & - & - & + & + & + \\
\hline
\end{tabular}

Control

$\gamma$-irradiation (5 Gy, 2 h)

PARPi $(10 \mu \mathrm{M})$

SAHA $(5 \mu M)$

Chloroquine diphosphate $(16 \mu \mathrm{M})$

Vitamin D2 (100 nM)

Lisinopril (100 nM)

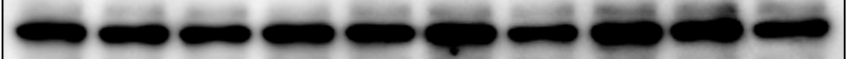

ACE2 (97 kDa)

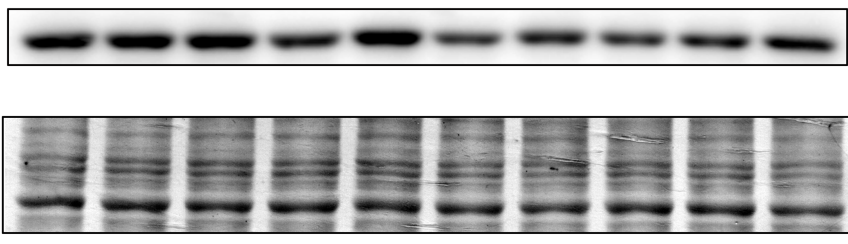

\section{Renin ( 45 kDa)}

Total

protein

level

$\begin{array}{llllllllll}1 & 2 & 3 & 4 & 5 & 6 & 7 & 8 & 9 & 10\end{array}$

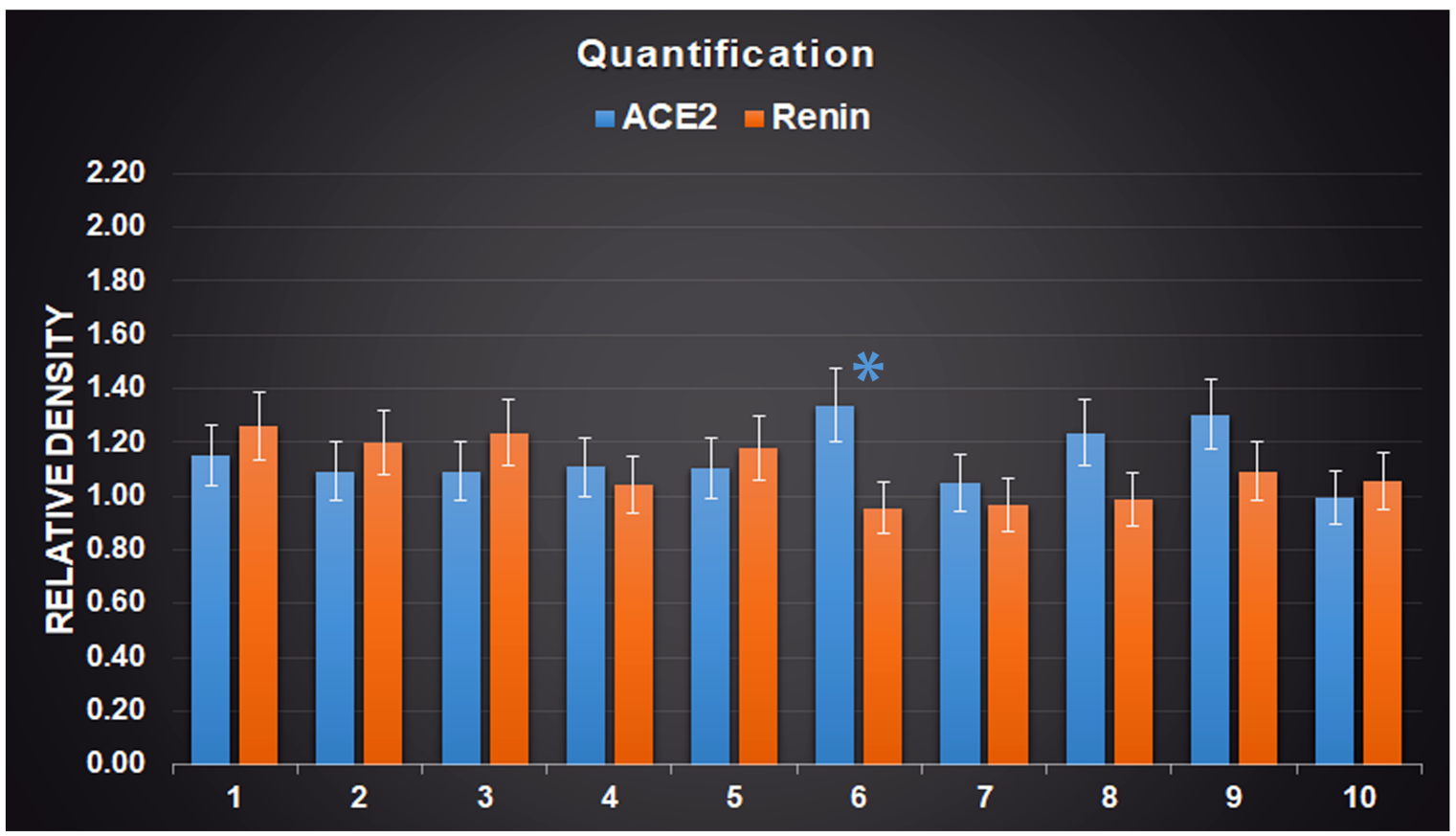

Figure 4

ACE2 and renin levels after distinct treatments of A549 cells. Effect of following drugs were analyzed: vitamin D2, ACE inhibitor Lisinopril, PARP inhibitor (olaparib), HDAC inhibitor (SAHA), y-irradiation, and chloroquine (antimalarial chloroquine diphosphate) or their combination. Data were normalized to the total protein levels, as shown in the bar chart. Quantification of the protein levels, studied by western 
blots, was performed by ImageJ software. The asterisk indicates a slight increase in the ACE2 level in the cells treated by vitamin D2.
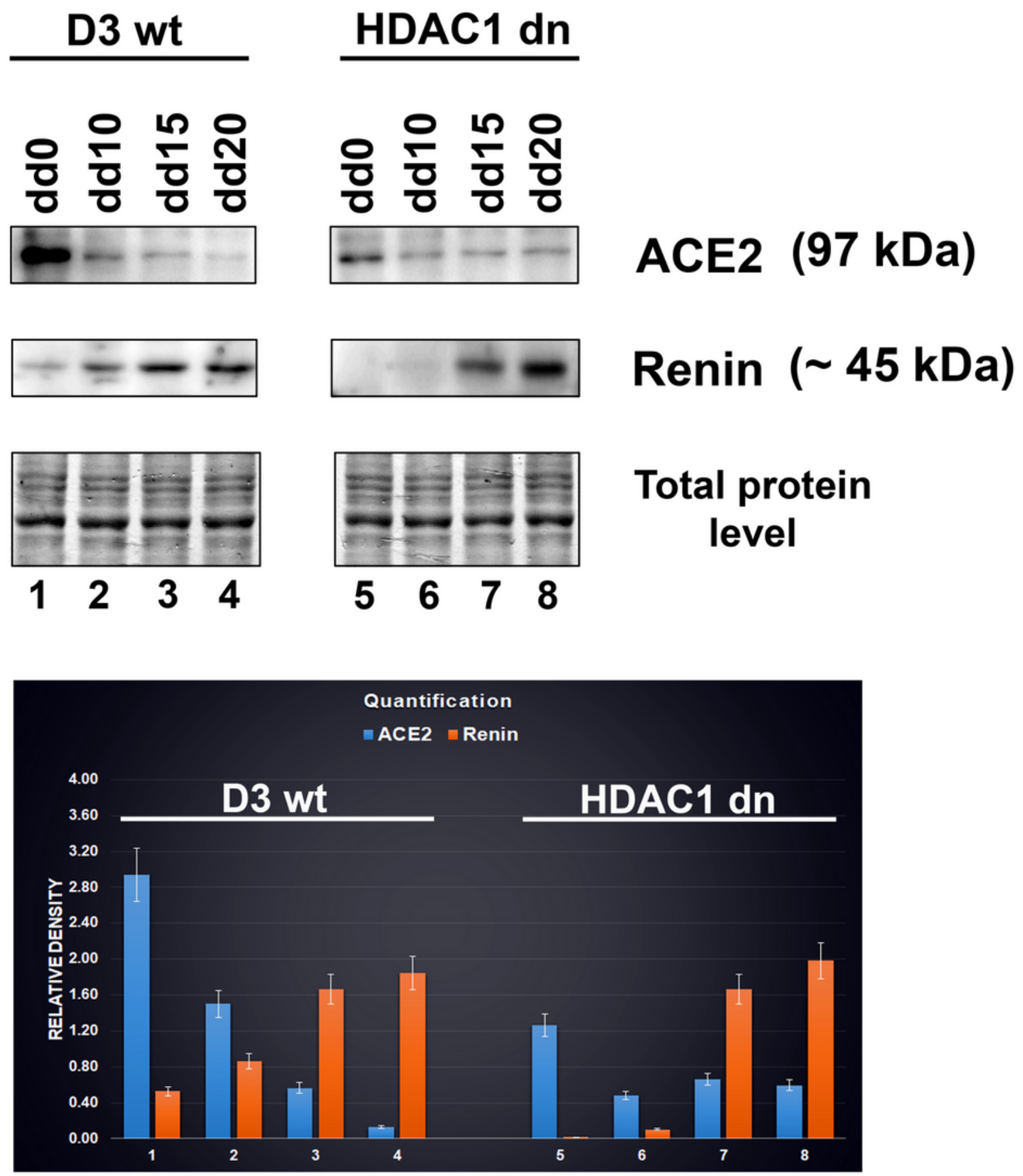

Figure 5

Down-regulation of ACE2 in mESCs undergoing differentiation into cardiomyocytes. Western blot analyses were performed in HDAC1 wt and HDAC1 depleted (dn) mES cells, non-differentiated, and differentiated into cardiomyocytes. Data show the levels of the ACE2 protein and renin normalized to the 
total protein levels. Quantification of the protein levels, studied by western blots, was performed by ImageJ software.

\section{ACE2 / $\alpha$-actinin / DNA}

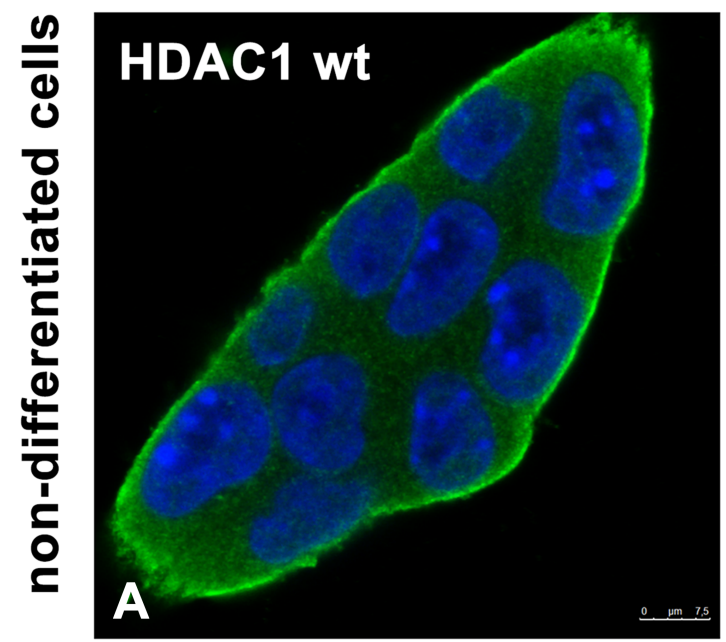

\section{HDAC1 dn}

\section{B}
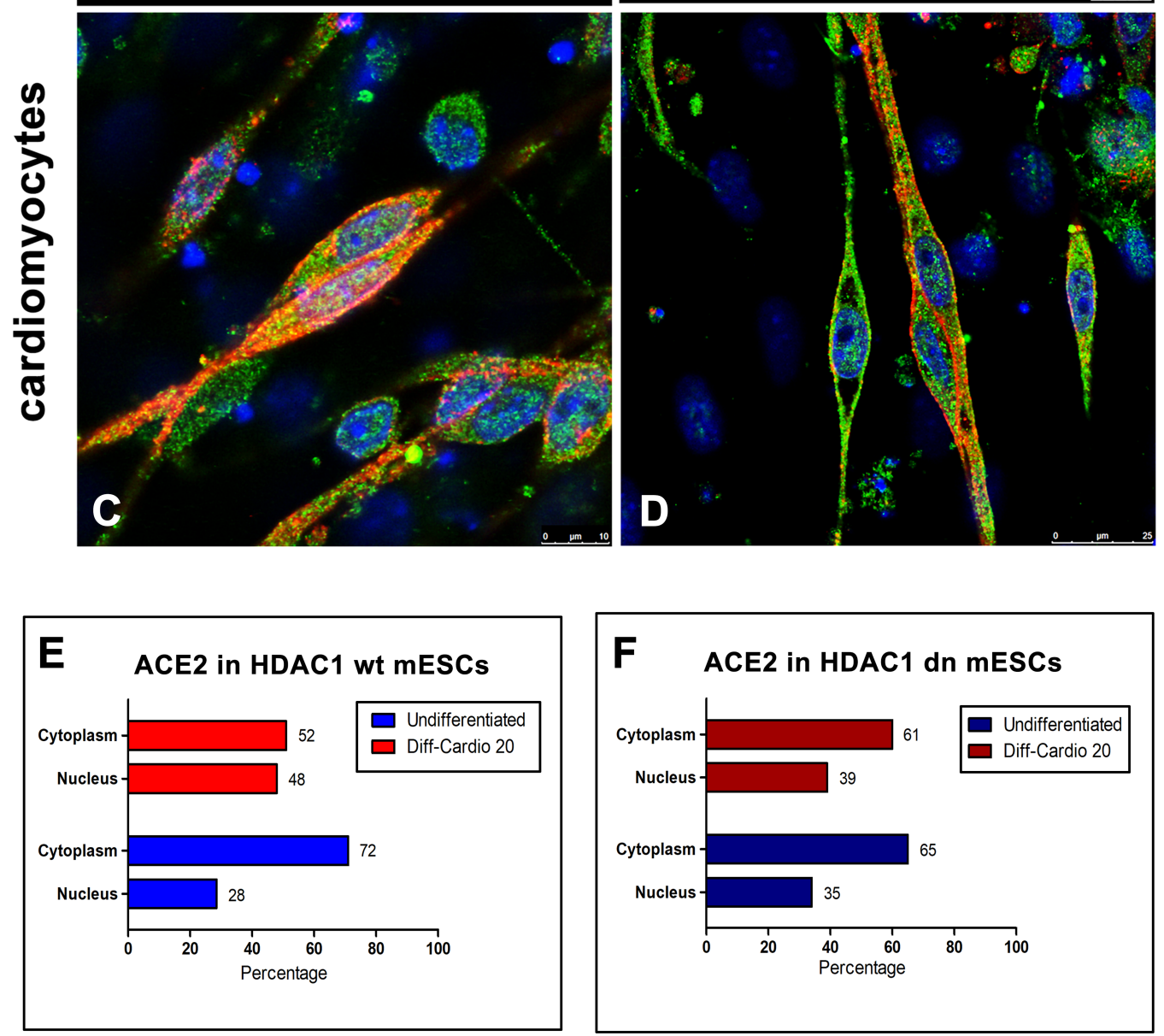

Figure 6

Distribution of ACE2 (green) in wt and HDAC1 depleted mES cells, non-differentiated and differentiated into cardiomyocytes. Immunofluorescent analyses were performed in (A) HDAC1 wt mESCs; (B) HDAC1 dn mESCs; (C) in a-actinin (red) positive cardiomyocytes generated from HDAC1 wt mEScs; (D) a-actinin 
(red) positive cardiomyocytes generated from HDAC1 dn mESCs. DAPI (blue) was used as a counterstaining. (E) Nucleo/cytoplasmic ration of the level of ACE2 is shown for HDAC1 wt mESCs and (F) Nucleo/cytoplasmic ration of the level of ACE2 is shown for HDAC1 dn mESCs.
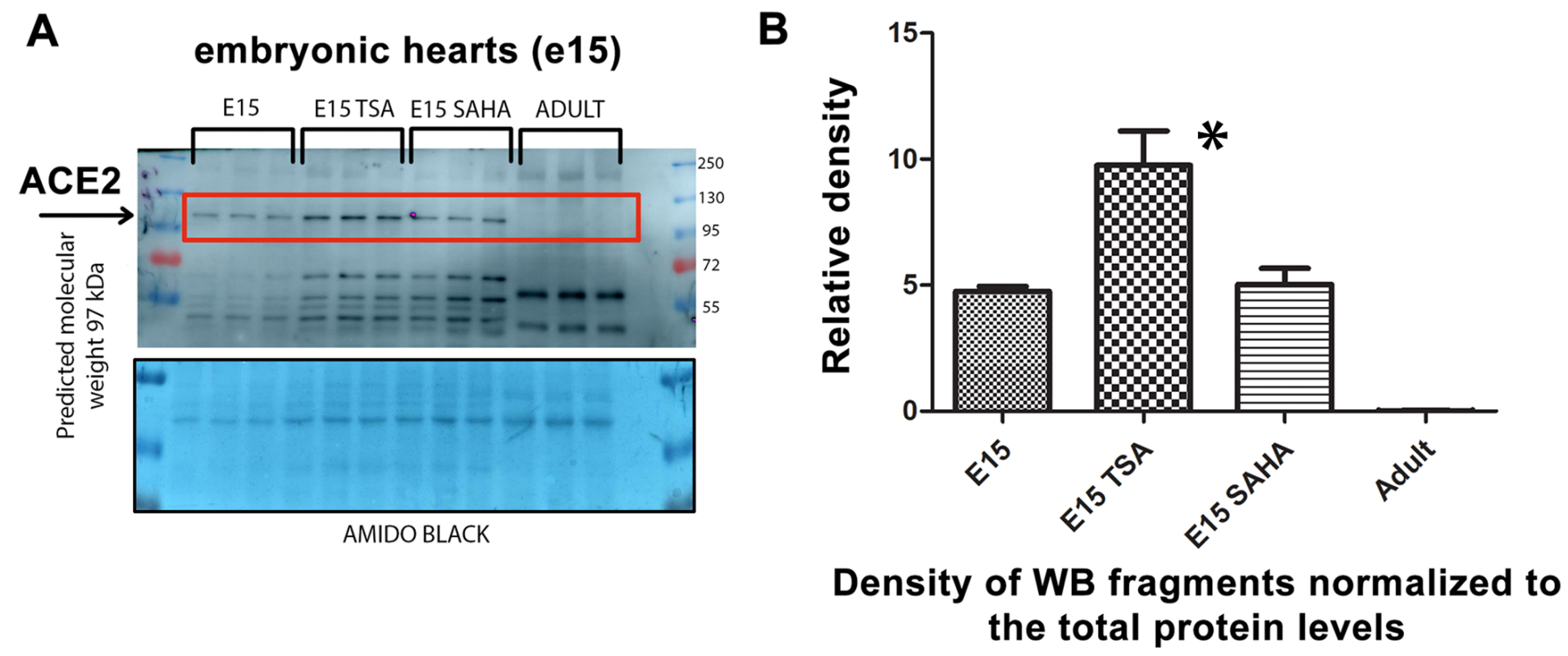

Figure 7

The level of the ACE2 protein studied in explanted embryonic hearts treated by HDAC inhibitors. (A) In non-treated, TSA (Trichostatin A)-, VPA (valproic acid)-, and SAHA (suberoylanilide hydroxamic acid)treated explanted mouse hearts at stage e15, the ACE2 protein level was analyzed. (B) Quantification of the protein levels, studied by western blots, was performed by ImageJ software. 